\title{
Editorial
}

\section{Diagnostic techniques in suspected thoracic aortic dissection}

Although mortality in thoracic aortic dissection remains high, early accurate diagnosis, particularly in patients with a type A dissection, can lead to life-saving surgical intervention. Several imaging techniques can now establish the diagnosis. The availability and usefulness of these techniques vary with different centres.

\section{Transthoracic echocardiography}

After presenting at a district general hospital the patient with a suspected dissection should undergo transthoracic echocardiography. Most district hospitals now have either a technician or a cardiologist capable of performing a thorough echocardiographic examination. The advantages of echocardiography are the speed and relative ease with which the technique can be used in a haemodynamically compromised patient. Reports of the accuracy of the method vary. The key echocardiographic sign is the detection of a flap of intima and media within the lumen of the aorta. Different studies have shown a sensitivity of between $14 \%$ and $100 \% .^{1-3}$ Other findings such as aortic root dilatation, thickening of the aortic wall, and evidence of a pericardial effusion or aortic regurgitation are nonspecific. The studies indicated that transthoracic echocardiography is limited in its usefulness ${ }^{1-3}$; though if skilled operators are available, it certainly has a role in triage at the district general hospital.

\section{Computed tomography}

Computed tomography has had a considerable impact on the management of patients with suspected dissection, both in the district general hospital and in the tertiary referral centre. The detection of a flap establishes the diagnosis and the anatomical extent of the dissection can be determined. Good quality, high density images that do not show a flap imply that dissection is extremely unlikely. However, poor quality images with inadequate opacification and movement and streak artefact are not uncommon and cannot securely exclude a diagnosis of dissection. ${ }^{4}$ In addition, computed tomography gives no information on left ventricular function or aortic regurgitation-both important determinants of survival. In addition the images require interpretation by an experienced radiologist. Patients with a negative or inadequate CT scan in whom there is continuing doubt over the clinical diagnosis should undergo coronary and aortic angiography. ${ }^{5}$

\section{Transoesophageal echocardiography}

Upon transfer to the referral centre the patient and any available investigations should be reviewed by a cardiologist, a cardiothoracic surgeon, and a radiologist. If all are satisfied with the diagnosis and information provided, the appropriate course of management can be started. If not, other diagnostic techniques should be used. Transoesophageal echocardiography is becoming increasingly popular because unlike transthoracic echocardiography, it is not affected by the structure of the thoracic cage or by respiratory movements. It provides high quality images of both the ascending and descending aorta, although the trachea may obscure part of the ascending aorta and arch. ${ }^{6}$ In several series sensitivities and specificities of up to $99 \%$ were reported: these are as good as both aortography and computed tomography. ${ }^{7-9}$ In centres with experienced operators there is no doubt that transoesophageal echocardiography can provide rapid and accurate diagnostic information. Unless performed expeditiously and with adequate sedation, transoesophageal echocardiography can increase systolic blood pressure. The main limitation in the use of this technique is the lack of appropriately trained operators. The provision of an adequate transoesophageal echocardiography service requires not only the appropriate equipment but also at least three experienced operators. Currently few centres are in a position to provide such a service, but it is likely that as experience with the technique becomes more widespread it will be used more often in patients with suspected dissection.

\section{Intravenous digital subtraction angiography}

Intravenous digital subtraction angiography has found favour in some centres and has obvious advantages. It is quick and easy to perform and only minimally invasive. Furthermore, its sensitivity and specificity are said to approach that of aortography. ${ }^{10}$ However, the equipment is not always available and the sensitivity and specificity are little better than with computed tomography, which is more widely available. Most surgeons would not operate solely on the basis of a digital subtraction angiogram but digital angiography may be a useful screening procedure.

\section{Magnetic resonance imaging}

A recent study suggests that magnetic resonance imaging has $100 \%$ specificity and sensitivity in diagnosing the presence of dissection compared with the reference diagnostic techniques of angiography, surgery, or necropsy. ${ }^{11}$ Magnetic resonance imaging was better than transoesophageal echocardiography, with fewer false positive diagnoses of dissection in the ascending aorta. Transoesophageal echocardiography has the advantage of taking less time than magnetic resonance imaging, though in the population studied no complications arose from the time spent in the scanner.

\section{Aortography}

Traditionally aortography has been accepted as the reference standard against which newer diagnostic techniques should be compared. There is considerable justification for this belief. Several very large multicentre studies have been performed and have shown sensitivities between $90 \%$ and $98 \% .^{12}{ }^{13}$ In addition, and perhaps of even more importance, aortography has been available since 1949 and consequently both cardiologists and cardiothoracic 
surgeons have developed considerable confidence in it. The aortogram provides information on the presence and severity of associated aortic regurgitation and, if it is negative, it may be useful to proceed to coronary angiography to investigate alternative diagnoses. Aortography can give false negative results, however, ${ }^{614}$ and of the diagnostic techniques available it carries the highest risk. In the future it is likely to be superseded by the newer imaging techniques.

\section{Coronary angiography}

Is coronary angiography necessary in patients in whom the diagnosis of dissection has been established? There is no published evidence that performing coronary angiography lowers mortality in patients with dissection. ${ }^{15}$ Most cardiologists and cardiothoracic surgeons think that coronary angiography in the presence of a very dilated ascending aorta substantially lengthens the procedure thereby exposing the patient to extra risk. In the absence of evidence to the contrary there seems no reason to perform coronary angiography routinely in patients in whom the diagnosis of dissection has been established.

\section{Conclusions}

We believe that if a good quality, high resolution computed tomography scan shows a dissection then no other investigation is necessary. A negative high quality computed tomographic scan in the district general hospital will usually avoid an inconvenient and costly transfer to a regional centre. If, however, no scan is available, the scan is non-diagnostic, or sufficient clinical suspicion remains despite a negative scan the patient should be transferred without delay. At the referral centre, in future, transoesophageal echocardiography is likely to be performed as the next diagnostic procedure. Magnetic resonance scanning will be increasingly available and useful in this setting with angiography being used largely to investigate the coronary arteries in patients in whom doubts about the diagnosis persist.

JONATHAN CLAGUE* PATRICK MAGEE $\dagger$ PETER MILLS ${ }^{\star}$

Cardiac Department ${ }^{\star}$ and Department of Cardiothoracic Surgery, $\dagger$

Royal London Hospital NHS Trust,

Whitechapel Road, London E1 $1 B B$

1 Granto DE, Dee P, Gibson RS. Utility of two-dimensional echocardiography in suspected ascending aortic dissection. Am $J$ Cardiol 1985;56:123-9.

2 Smackler AR, Rosmir AM, Watts LE, Hackshaw BT. Echocardiographic diagnosis of aortic root dissection by $\mathrm{M}$-mode and two-dimensional techniques. Am Heart J 1982;103:897-904.

3 McLeod AA, Monaghan MJ, Richardson PJ. Diagnosis of acute aortic dissection by $\mathrm{M}$-mode and cross-sectional echocardiography: a five year experience. Eur Heart $J$ 1983;4:196-202.

4 Vassile N, Mathieu D, Keita K, et al. Computed tomography of thoracic aortic dissection: accuracy and pitfalls. J Comput Assist Tomogr 1986; 10:211-5.

5 Morgan JM, Oldershaw PJ, Gray HH. Use of computed tomographic scanning and aortography in the diagnosis of acute dissection of the scanning and aortography in the diagno
thoracic aorta. Br Heart $J 1990 ; 64: 261-5$.

6 Mugge A, Daniel WG, Laas J, Grote R, Litchtlen PR. False-negative diagnosis of proximal aortic dissection by computerised tomography or angiography and possible explanations based on transoesophageal echocardiographic findings. Am J Cardiol 1990;65:527-8.

7 Erbel R, Engberding R, Daniel W, Roelandt J, Visser C, Rennollet H. Echocardiography in diagnosis of aortic dissection. Lancet 1989;i:457-61

8 Enia F, Ledda G, Lo Maura R, Matassa C, Raspanti G, Stabile A. Utility of echocardiography in the diagnosis of aortic dissection involving the ascending aorta. Chest 1989;95:125-9.

9 Godwin JD, Herfkens RL, Skioldebrand CG, Ferdele MP, Lipton MJ. Evaluation of dissections and aneurysms of the thoracic aorta by conventional and dynamic CT scanning. Radiology 1980;136:125-33.

10 Lyons JL, Gershlick A, Gardener J, Layton C. Arrival time analysis of intravenous digital aortograms in aortic dissection. Br Heart J 1988; 166:651-5.

11 Nienaber CA, Spielmann RP, von Kodolitsch Y, et al. Diagnosis of thoracic aortic dissection: magnetic resonance imaging versus transesophageal aortic dissection: magnetic resonance imaging

12 Slater EE, De Sanctis RW. The clinical recognition of dissecting aortic aneurysm. Am J Med 1976;60:625-33.

13 Smith DC, Jang CC. Radiological diagnosis of aortic dissection. In: Doroghazi RM, Slater EE, eds. Aortic dissection. New York: McGrawHill, 1983:71-132.

14 Shuford WH, Sybers RG, Weens HS. Problems in aortographic diagnosis of dissecting aneurysm of the aorta. $N$ Engl J Med 1969;280:225-31.

15 Kern MJ, Serota H, Callicoat $P$, et al. Use of coronary arteriography in the preoperative management of patients undergoing urgent repair of the thoracic aorta. Am Heart J 1990;119:143-8. 